\title{
The circadian molecular clock creates epidermal stem cell heterogeneity
}

Peggy Janich ${ }^{1}$, Gloria Pascual ${ }^{1}$, Anna Merlos-Suárez ${ }^{2}$, Eduard Batlle ${ }^{2,3}$, Jürgen Ripperger ${ }^{4}$, Urs Albrecht ${ }^{4}$, Karl Obrietan $^{5}$, Luciano Di Croce ${ }^{1,3}$ \& Salvador Aznar Benitah ${ }^{1,3}$

\begin{abstract}
Murine epidermal stem cells undergo alternate cycles of dormancy and activation, fuelling tissue renewal. However, only a subset of stem cells becomes active during each round of morphogenesis, indicating that stem cells coexist in heterogeneous responsive states. Using a circadian-clock reporter-mouse model, here we show that the dormant hair-follicle stem cell niche contains coexisting populations of cells at opposite phases of the clock, which are differentially predisposed to respond to homeostatic cues. The core clock protein Bmall modulates the expression of stem cell regulatory genes in an oscillatory manner, to create populations that are either predisposed, or less prone, to activation. Disrupting this clock equilibrium, through deletion of Bmal1 (also known as Arntl) or Per1/2, resulted in a progressive accumulation or depletion of dormant stem cells, respectively. Stem cell arrhythmia also led to premature epidermal ageing, and a reduction in the development of squamous tumours. Our results indicate that the circadian clock fine-tunes the temporal behaviour of epidermal stem cells, and that its perturbation affects homeostasis and the predisposition to tumorigenesis.
\end{abstract}

Epidermal stem cells ensure that skin homeostasis is maintained. Murine epidermal stem cells are located either at the permanent portion of the hair follicle-termed the bulge-and are exclusively responsible for hair cycling ${ }^{1-4}$; or at the junction between the epidermis and the hair follicle (isthmus), and feed into the epidermis and sebaceous glands ${ }^{5-7}$. In addition, a continuous proliferation of basal interfollicular epidermal cells ensures daily epidermal maintenance ${ }^{8}$.

Bulge stem cells undergo bouts of activation followed by periods of dormancy, to establish hair follicle cycling. Robust TGF- $\beta$ and Bmp signals act as 'activation breaks', rendering bulge cells dormant during the resting phase of the hair cycle (telogen $)^{9-11}$. At the onset of the growth phase (anagen), bulge cells respond to Wnt signals by migrating into the lower proliferative hair germ region, where they contribute to follicle growth ${ }^{12-15}$. Subsequently, at mid-anagen, the bulge undergoes a second round of activation, which replenishes cells lost at the onset of anagen ${ }^{2,3}$. However, the response of bulge stem cells to activating stimuli is a heterogeneous process, as only a subset of them become active during either stage of activation ${ }^{12,13}$. The nature of such niche heterogeneity is currently unknown. Importantly, perturbing the equilibrium between the responsive and non-responsive stem cell states causes tissue malfunction and increases the risk of carcinogenesis ${ }^{16-20}$.

Here, we analysed the role of the molecular clock in fine-tuning the function of epidermal stem cells. The mammalian clock machinery anticipates and synchronizes vital functions related to the physiological circadian needs of the organism ${ }^{21}$. The core molecular clock is established by a positive limb, composed of heterodimers of the transcription factors Clock and Bmal, which drives the rhythmic expression of the negative limb proteins, period (Per1-3), cryptochrome (Cry1/2), and Dec1/2 (ref. 21). Accumulated Per and Cry proteins in turn translocate to the nucleus and inhibit Bmal/Clock transcriptional activity, thereby repressing their own expression and marking the beginning of a new cycle.

\section{Circadian heterogeneity in hair-follicle stem cells}

We monitored the activity of the clock in epidermal stem cells by means of a reporter bacterial artificial chromosome (BAC) transgenic mouse, in which the expression of fluorescent venus is under the regulation of the full-length promoter of the Per1 gene ${ }^{22}$. Venus expression mirrors the endogenous oscillation of the clock in the suprachiasmatic nuclei, where the central pacemaker resides, thereby establishing its bona fide circadian reporter activity ${ }^{22}$. We first studied the behaviour of the clock in the dorsal skin of Per1-venus mice collected between postnatal days (P)19 and P31, when hair follicles synchronously transit from the dormant to the growth phase.

At P19, a stage in which the bulge is predominantly dormant, the bulge cells expressing CD34 and the highest levels of $\alpha 6$ integrin ( $\alpha 6$ integrin ${ }^{\text {bright }} / \mathrm{CD} 34^{+}$) contained a continuum of venus-expressing populations, which could be subdivided into cells with highest mean fluorescence intensity of venus (venus ${ }^{\text {bright }}$ ), and those with no venus fluorescence (venus ${ }^{\mathrm{dim}}$ ), as determined by immunohistochemistry (Supplementary Fig. 1), and fluorescence-activated cell sorting (FACS) (Fig. 1a, b). At this stage, the bulge contained approximately equal numbers of venus ${ }^{\text {bright }}$ and venus ${ }^{\text {dim }}$ stem cells (Fig. 1a, b). As hair follicles synchronously progressed into anagen (from P20 to P31), the proportion of venus ${ }^{\text {bright }}$ bulge cells steadily increased, reaching a proportion of $90 \%$ venus ${ }^{\text {bright }}$ to $10 \%$ venus ${ }^{\mathrm{dim}}$ at the peak of follicle growth (Fig. 1a, b). Conversely, the basal layer of the interfollicular epidermis ( $\alpha 6$ integrin ${ }^{\text {bright }} / \mathrm{CD} 34^{-}$) showed a homogenous pattern of clock activity with a ratio of 95:5 venus ${ }^{\text {bright }}$ :venus ${ }^{\text {dim }}$ cells, irrespective of the day analysed (Supplementary Fig. 2). Heterogeneity of the clock phase in bulge cells during the telogen to anagen transition was also observed with another independently generated reporter line, Per1-GFP, as shown by three-dimensional wholemount GFP fluorescence imaging of tail epidermis (Supplementary Fig. 3a, b) ${ }^{23}$.

We next verified whether these clock stem cells states showed circadian rhythmicity. FACS analysis indicated that the expression of venus in bulge stem cells in anagen (P27), or interfolllicular epidermis basal cells, followed a circadian pattern, irrespective of whether the mice were maintained in $12 \mathrm{~h}$ light/dark cycles, or in constant darkness under free running conditions (Fig. 1c and Supplementary Fig. 4a, b) ${ }^{21}$. This circadian variation was further confirmed 

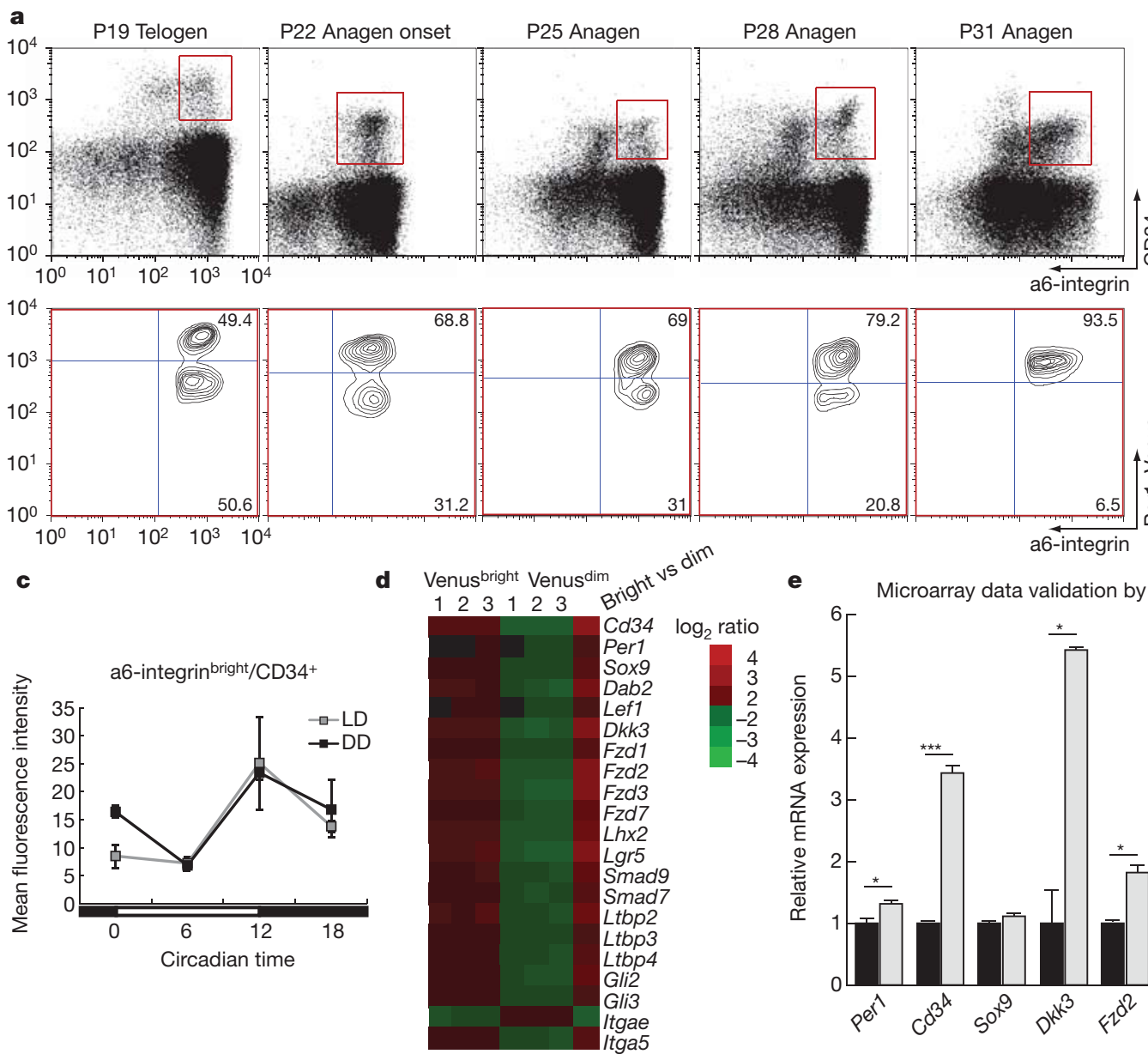

Figure $1 \mid$ The molecular clock regulates the expression of the bulge stem cell signature. a, b, Percentages of venus ${ }^{\text {bright }}$ and venus ${ }^{\text {dim }}$ cells in the bulge by FACS $(n \geq 6)$. c, Venus mean fluorescence intensity of bulge cells in mice kept under $12 \mathrm{~h}$ light $/ 12 \mathrm{~h}$ dark (LD) and $12 \mathrm{~h}$ dark $/ 12 \mathrm{~h}$ dark (DD) conditions $(n=2)$. d. Heatmap of selected genes from arrays of venus ${ }^{\text {bright }}$ and venus ${ }^{\mathrm{dim}}$

by time-lapse in vivo confocal microscopy of venus fluorescence in dorsal skin explants biopsied from adult Per1-venus mice, and GFP fluorescence in whole mounts of tail epidermis of Per1-GFP mice (Supplementary Fig. 4c, d).

\section{The clock regulates stem cell genes}

We then compared the global transcriptomes of purified venus ${ }^{\text {bright }}$ and venus ${ }^{\mathrm{dim}}$ bulge cells from the dorsal skin of P18-19 mice ( $\alpha 6^{\text {bright }}$ / $\mathrm{CD} 34^{+} /$venus $^{\text {bright }}$ and $\alpha 6^{\text {bright }} / \mathrm{CD} 34^{+} /$venus $^{\text {dim }}$ ). As expected, both populations showed differential expression of core circadian transcripts, such as Cry2, Per1, Nr1d1, Ror $\beta, D e c 2$ and E4BP4 (also known as Nfil3) (Fig. $1 \mathrm{~d}$ and Supplementary Table 1). Intriguingly, although the bulge is inactive at P18-19, both populations differed in the expression of a significant number of genes previously shown to constitute the bulge signature ${ }^{2,3,12,24}$. Venus ${ }^{\text {bright }}$ bulge stem cells expressed higher levels (between 1.4- to 3-fold) of Wnt-signalling factors, including Tcf3, Fzd2/3, Sox9, Lhx2, Lgr5, Lef1, Dkk3 and $D a b 2$, as well as TGF- $\beta$-inhibitory factors such as $S m a d 7, L t b p 2-4$, Smurf, Lefty and Cul1 (Fig. 1d, e, and Supplementary Table 1). Other pathways differentially expressed relevant for bulge behaviour related to integrins, Notch, Bmp and Shh, among others (Fig. 1d, e).

Thus, the coexisting clock states of dormant bulge stem cells correlated with differential expression of key epidermal homeostasis genes. Promoter analysis revealed that several of these genes, encoding for proteins known to control bulge dormancy, activation, differentiation and niche interactions ${ }^{1}$, contained several putative Bmal1/

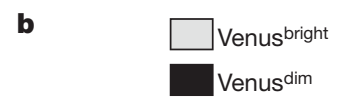

Venus dim
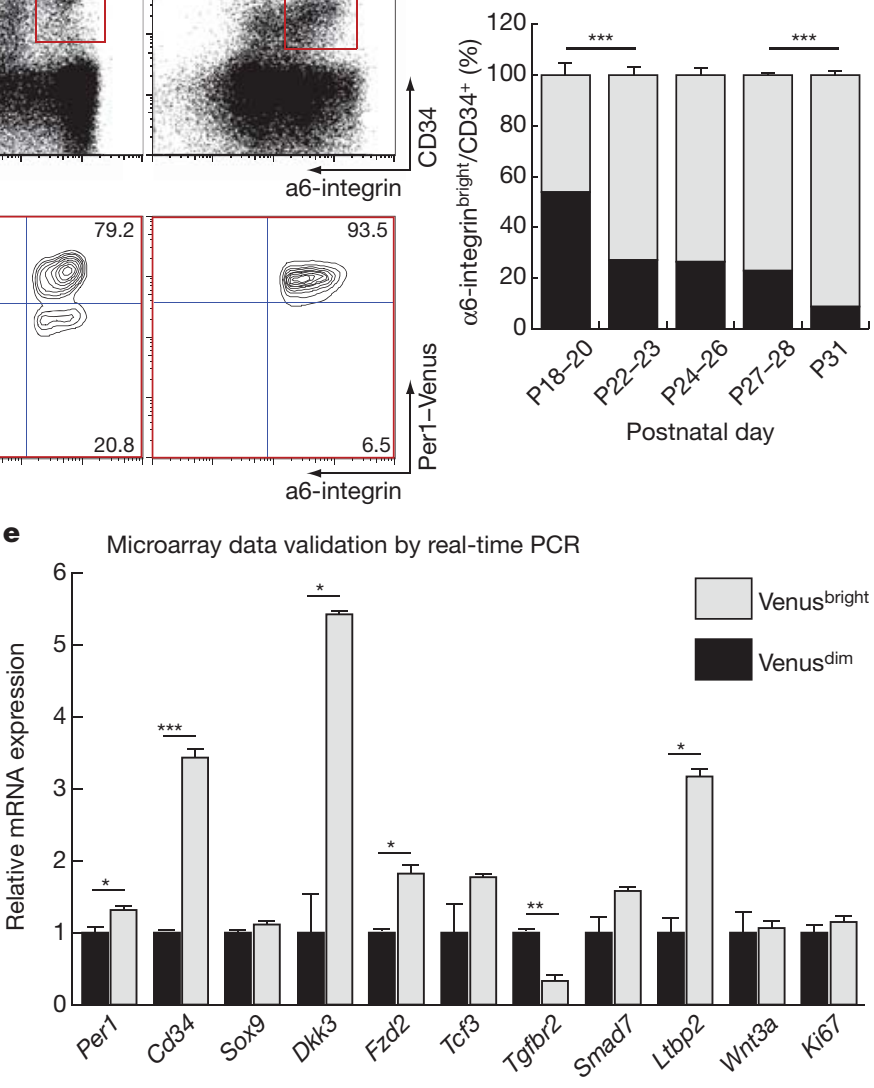

bulge cells of P19 mice $(n=3)$. e, Validation of microarray data by real-time PCR. Fold change is shown as relative to venus ${ }^{\text {dim }}$ cells after normalization to pumilio 1 (Pum1) ( $n=2$, pool of 6 mice per replicate). Results in $\mathbf{b}, \mathbf{c}$ and $\mathbf{e}$ are shown as mean \pm s.e.m., ${ }^{*} P<0.05,{ }^{* *} P<0.01, * * * P<0.001$ (two-tailed Student's $t$-test).

Clock-binding sites within their proximal and distal promoter regions (Supplementary Table 2). These included the Wnt signalling factors Dab2, Lef1, Dkk3, Fzd2, Sox9, Lhx2 and Tcf4; TGF- $\beta$ regulators such as Smad7, Lefty, Smurf2 and Smad9; and Itga6 as well as modulators of Bmp and Notch signalling (Supplementary Table 2). We confirmed by chromatin immunoprecipitation (ChIP) that Bmal1/Clock bound to these promoters in intact adult tail epidermis (Supplementary Fig. 5a), and that the binding of Bmall was circadian (Fig. 2). Chromatin occupancy of Bmall to these genes was also confirmed in FACS-sorted bulge stem cells (Supplementary Fig. 5b).

\section{Clock arrhythmia affects homeostasis}

We next sought to study the biological significance of this clock mechanism in epidermal stem cells in vivo. To this end, we generated mice with a conditional deletion of Bmal1 in the keratin- $14^{+}$basal keratinocyte compartment (K14Cre/Bmal1 ${ }^{\text {LoxP/LoxP }}$, hereafter refered as Bmal1KO) (Supplementary Fig. 6a, b). Deletion of Bmal1 causes circadian arrhythmicity without the need to perturb any other core circadian member ${ }^{21}$, and its ubiquitous deletion causes premature ageing, including defects in adult skin morphogenesi ${ }^{25-27}$. We crossed Bmal1KO and Per1-venus mice, and verified that the circadian clock of bulge stem cells and basal interfollicular epidermal cells was arrhythmic, and permanently skewed towards a clock ${ }^{\text {low }}$ (venus ${ }^{\text {dim }}$ ) state (Supplementary Fig. 6c).

Bulge stem cells and primary keratinocytes isolated from the dorsal skin of Bmal1 KO mice expressed lower transcript levels of Wnt-related 


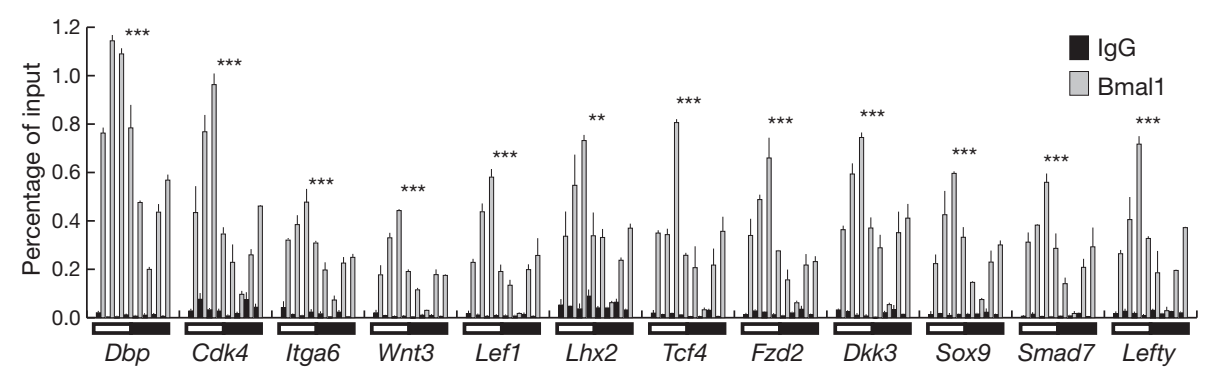

Figure $2 \mid$ Circadian binding of $\mathrm{Bmal}$ to the promoters of genes involved in adhesion, cell cycle, TGF- $\beta$ and Wnt pathways. ChIP from tail epidermis of wild-type mice collected every $3 \mathrm{~h}$ during $24 \mathrm{~h}$ (white and black bars represent day and night, respectively). Graph shows percentage of immunoprecipitated DNA over an input control from one representative experiment $(n=2)$.

genes including Dab2, Dkk3, Lef1 and Wnt10a, than control stem cells (Fig. 3a and Supplementary Fig. 5c). In addition, they contained lower messenger RNA levels of TGF- $\beta$ inhibitors, and higher amounts of Tgfbr 2 and Smad3 (Fig. 3a and Supplementary Fig. 5c). The expression of Wnt and TGF- $\beta$-related factors in purified bulge stem cells varied within a $12 \mathrm{~h}$ period in wild-type mice, but not Bmal1KO mice (Supplementary Fig. 5d). Accordingly, the hair follicles of Bmal1KO mice showed the same differences at the protein level, as exemplified by immunohistochemical analysis of Sox9, Lef1, phospho-Smad2 and TGF $\beta R 2$ in dorsal skin sections, and western blot from primary keratinocytes (Fig. 3b, d).

Altogether, these results indicated that the clock machinery might endow subpopulations of epidermal stem cells with different predispositions to respond to dormancy or activation stimuli, such as

a

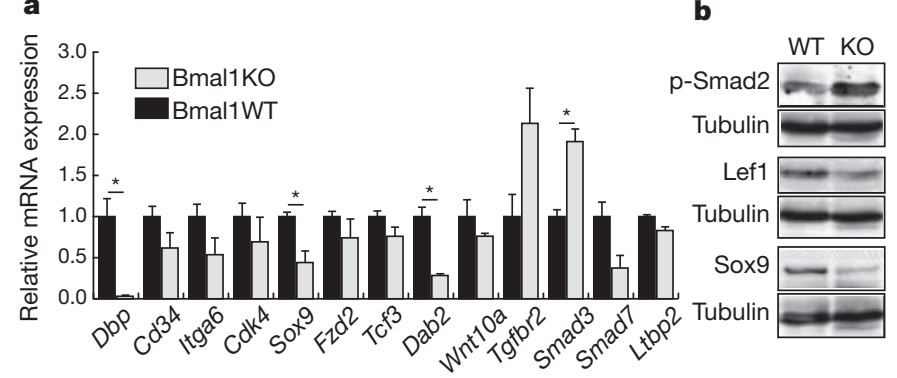

c
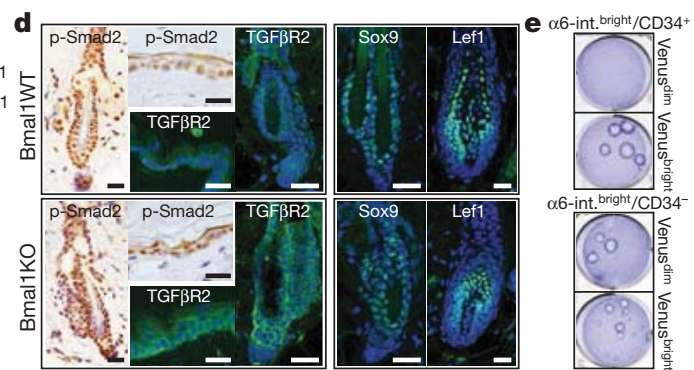

Figure $3 \mid$ Bmall modulates the response of bulge stem cells to activation and dormancy cues. a, Differential expression of genes in bulge cells of Bmal1WT and BmallKO mice. Fold-change values are shown as relative expression to Bmal1WT cells after normalization to Pum $1(n=2)$. b. Western blot analysis for phospho-Smad2 (p-Smad2), Lef1 and Sox9 in tail epidermis of 9-month-old BmallWT and BmallKO mice; $n=3$ mice were analysed for each group; c, Primary mouse keratinocytes of Bmal1KO show enhanced responsiveness to TGF- $\beta 1$ after $48 \mathrm{~h}$ of treatment $(n=3)$. d, Immunostaining for phospho-Smad2, TGFßR2, Lef1 and Sox9 in back skin of Bmal1WT and Bmal1KO mice $(n \geq 5)$. Scale bars $25 \mu \mathrm{m}$. e, Clonogenic assay of FACS-purified venus $^{\text {bright }}$ and venus ${ }^{\mathrm{dim}}$ bulge and interfollicular epidermis (IFE) keratinocytes from the back skin of P19 Per1-venus mice $\left(7 \times 10^{3}\right.$ bulge and $1 \times 10^{5}$ epidermal cells). Results in $\mathbf{a}$ and $\mathbf{c}$ are shown as mean \pm s.e.m., ${ }^{*} P<0.05$ (twotailed Student's $t$-test).
Results are shown as mean \pm s.e.m., $* * P<0.01, * * * P<0.001$ (one-way ANOVA). Statistical significance determined by Cosinor analysis revealed a $P$ value of $P<0.05$ for Dbp and Cdk4, and $P<0.001$ for Itga6, Lef1, Smad7, Sox 9 and Tcf4.

TGF- $\beta$ and Wnt. In line with this, the hair follicle bulge and interfollicular epidermis of Bmal1KO mice contained higher levels of active phospho-Smad2 (Fig. 3b, d). Moreover, Bmal1KO keratinocytes were more responsive to TGF- $\beta$ treatment than control keratinocytes (Fig. 3c). We could not detect any Wnt activity using the Wnt-specific reporter containing binding sites for TCF/Lef proteins (TOP-Flash) in our primary cultures of mouse keratinocytes stimulated with the GSK3b inhibitor 6-bromoindirubin-3-oxime (BIO), or purified Wnt3a, in accordance with previous reports ${ }^{28}$; this prevented us from further studying the effect of Bmall deletion on Wnt responsiveness (Supplementary Fig. 7).

When plated at clonal density, Perl-venus ${ }^{\text {bright }}$ bulge stem cells and basal epidermal cells showed a higher growth potential than the corresponding venus ${ }^{\mathrm{dim}}$ population, further suggesting that the clock $^{\text {high }}$ state is more prone to become activated than the clock ${ }^{\text {low }}$ counterpart (Fig. 3e). Additional data confirmed this hypothesis. First, the hair follicle bulges of Bmal1KO mice, which are permanently locked in the clock ${ }^{\text {low }}$ state, contained fewer proliferative cells and a higher number of long-term label (BrdU)-retaining dormant stem cells (LRCs), from 10 months of age and onwards (Fig. 4a). Second, the epidermis of another model of circadian arrhythmia, Perl and Per2 double-mutant mice (Per1/2dKO), which lacks the negative limb of the molecular clock and is therefore locked in the clock ${ }^{\text {high }}$ state, showed the opposite effects; that is, enhanced bulge proliferation, reduced numbers of bulge LRCs and sustained expression of epidermal clock target genes ${ }^{29}$ (Fig. 4b and Supplementary Fig. 8). The hair follicles of Bmal1KO were less efficient in becoming active upon depilation (Supplementary Fig. 9a). Bmal1KO bulge cells were also less hyperprolifertive than wild-type bulge cells in response to treatment with the phorbol ester 12-O-tetradecanoylphorbol-13-acetate (TPA), thus delaying the entry of the hair follicles into anagen (Supplementary Fig. 9b). Lastly, epidermal stem cells purified from Bmal1KO and Per1/ $2 \mathrm{dKO}$ mice were less and more clonogenic, respectively, in vitro than wild-type cells (Supplementary Fig. 10a, b). Of note, deletion of Bmal1 or Per1/2 did not affect the proportion of bulge stem cells in adulthood compared to their controls (Supplementary Fig. 11a, b).

\section{Loss of Bmall induces epidermal ageing}

Reduced and enhanced proliferation, respectively, was also evident in the basal layer of the interfollicular epidermis of Bmal1KO and Per1/ $2 \mathrm{dKO}$ mice (Supplementary Fig. 12). Bmal1KO mice showed signs of inefficient epidermal self-renewal, with premature signs of ageing as early as 5 months of age, such as the accumulation of terminally differentiated cornified cells (Fig. 4c and Supplementary Fig. 9c). This was accompanied by increased expression of $\mathrm{p} 16$, which has been previously associated with increased epidermal ageing ${ }^{30}$, but not p19 or apoptosis (Supplementary Fig. 13). Because bulge stem cells do not contribute to epidermal maintenance in steady-state conditions $s^{31}$, we sought to understand the molecular mechanisms underlying the defects of the interfollicular epidermis of BmallKO mice. We performed 
a
Bmal1WT
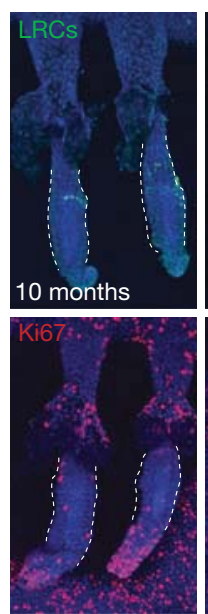

Bmal1KO
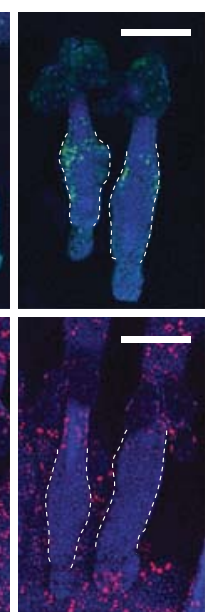

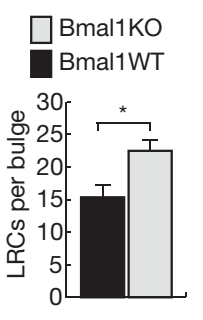

$\square$ Bmal1KO

Bmal1WT

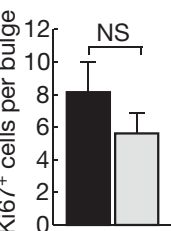

b

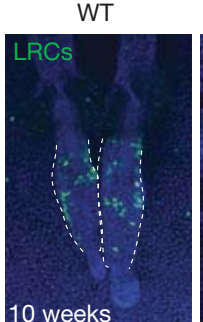

10 weeks
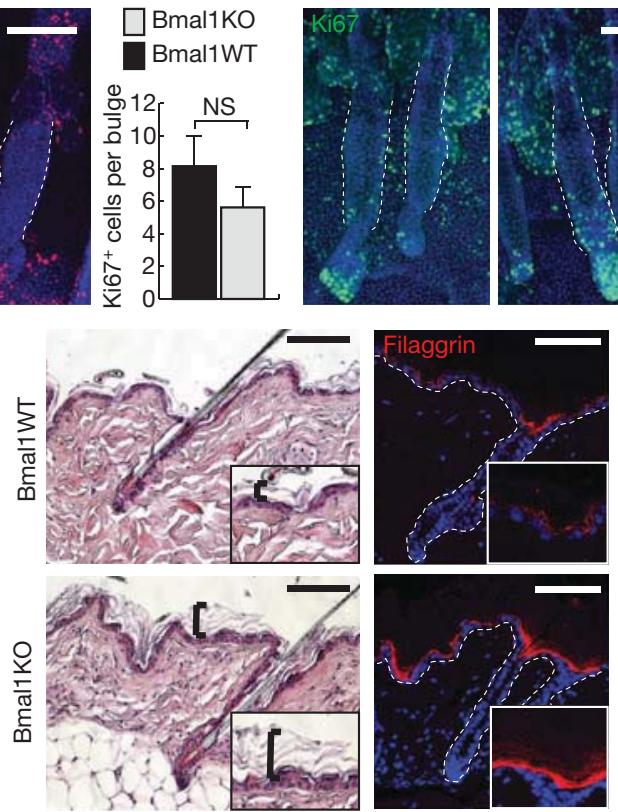

10-12 months
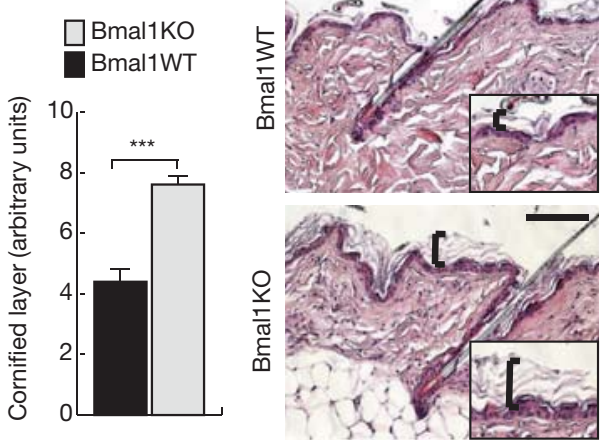

Per1/2dKO
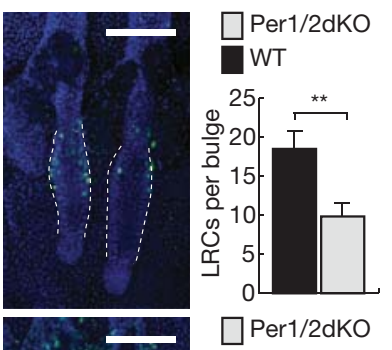

WT

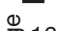

을 16

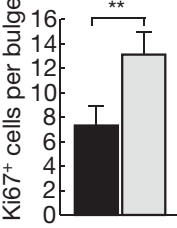

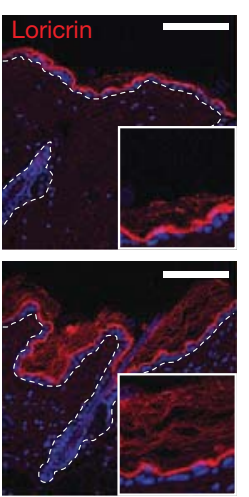

Figure 4 | Clock perturbation in vivo results in changes in the number of dormant bulge stem cells, and premature epidermal ageing.

a, b, Quantification of BrdU ${ }^{+}$LRCs and $\mathrm{Ki}^{+} 7^{+}$cells in the bulge of 10 -monthold Bmal1WT and Bmal1KO mice (a) and 10-week-old wild type (WT) and Per1/2dKO mice (b), showing opposite phenotypes $(n \geq 4 ; 9$ follicles per

mouse were analysed). c, Histological analysis and immunostaining for filaggrin and loricrin (red) in 10-12-months-old Bmal1WT and Bmal1KO mice. Graph shows thickness quantification of the cornified layer $(n=13)$. Scale bars, $100 \mu \mathrm{m}$. Results are shown as mean \pm s.e.m., ${ }^{*} P<0.05,{ }^{*} P<0.01$, $* * * P<0.001$ (two-tailed Student's $t$-test). NS, not significant.

microarray analysis of purified basal interfollicular epidermal progenitors ( $\left.\alpha 6^{\text {bright }} / \mathrm{CD} 34^{\text {neg }}\right)$, from 10-month-old Bmal1KO mice and their control littermates (Supplementary Table 3). As expected, cells from Bmal1KO mice showed strong differential expression of most of the core circadian transcripts, including the microRNA miRNA-122 (ref. 32), some of which were validated independently by real-time quantitative PCR with reverse transcription (RT-qPCR) (Supplementary Fig. 14a and Supplementary Table 3). Interestingly, Gene Ontology (GO) analysis indicated that the cell cycle, energy and drug metabolism, calcium-sensing proteins, the epidermal barrier response and chromatin compaction were significantly affected upon deletion of Bmal1 (Supplementary Fig. 14b). Intriguingly, although Bmal1KO mice showed a hyperkeratotic phenotype, the viable epidermal layers expressed lower levels of terminal differentiation markers, including Flg, Lor, Sprr1, Lce genes and Tgm (Supplementary Fig. 14c and Supplementary Table 3 ). Interestingly, Bmal1 KO cells also expressed lower amounts of the miRNA-23b/-27b/-24-1 cluster, which targets TGFßR2 and Smad proteins ${ }^{33}$ (Supplementary Fig. 14d). The reduced levels of epidermal differentiation genes probably reflects the lower efficiency of activation of basal interfollicular epidermal cells in Bmal1KO mice, suggesting that the hyperkeratotic phenotype developed as a compensatory mechanism to ensure a certain degree of epidermal barrier protection.

\section{Loss of Bmall reduces skin tumorigenesis}

Because perturbation of the clock machinery affects the predisposition of certain tissues to carcinogenesis ${ }^{34}$, we next studied whether

epidermal deletion of Bmall had any impact on the development of cutaneous squamous tumours. To this end, we crossed Bmal1KO mice with a transgenic line expressing oncogenic Sos, an activator of Ras, under the regulation of the Krt5 promoter (K5-SOS) ${ }^{35}$. In an EGFR mutant-heterozygous background, K5-SOS mice spontaneously developed squamous tumours, primarily in the tail, with $100 \%$ penetrance, as previously described ${ }^{35}$. Bmal1KO/K5-SOS mice developed significantly fewer neoplastic lesions at early-, mid- and late-stages of carcinoma development than control mice (Fig. 5a and Supplementary Fig. 15a). The skin lesions of Bmal1KO/K5-SOS mice were more differentiated-as determined by increased expression of involucrin and loricrin-contained large cornified islands and a higher number of apoptotic areas, as compared to control tumours (Supplementary Fig. 15b). Control mice had to be killed by two months of age, a time at which no Bmal1KO mice had developed the number, or size, of tumours. The growth of cutaneous squamous tumours has been shown to depend on Wnt activity in a population of $\mathrm{CD} 34^{+}$tumour-initiating cells ${ }^{36}$. However, we could not detect any nuclear $\beta$-catenin, either in control or in Bmal1KO neoplastic lesions, suggesting that, in our model, tumour growth did not primarily depend on misregulated Wnt signalling (Supplementary Fig. 16a, b). We did observe a significant reduction in the percentage of $\alpha 6^{\text {bright }} / \mathrm{CD} 34^{+}$tumour-initiating cells in BmalKO tumours with respect to wild-type tumours (Fig. 5b and Supplementary Fig. 17). Notably, whereas wild-type tumours expressed $\alpha 6$ integrin in basal and suprabasal cells, which has been previously associated with increased malignancy ${ }^{37}$, Bmal1KO tumours only expressed it in basal 


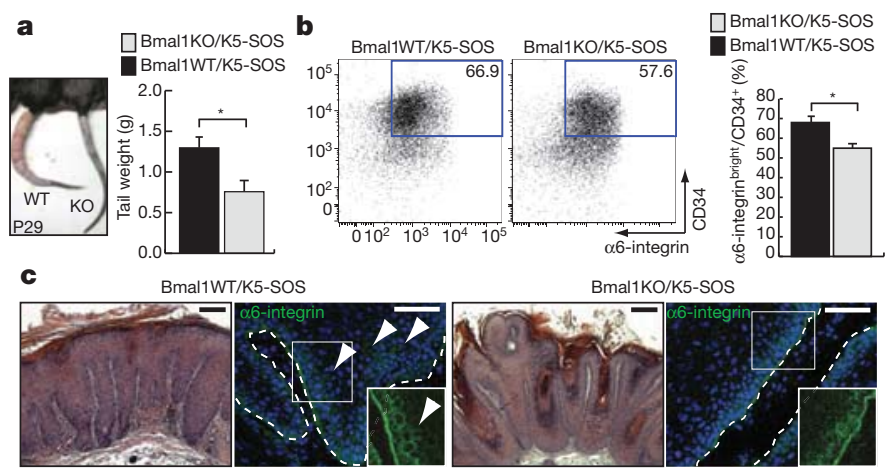

Figure $5 \mid$ Loss of Bmal1 reduces the development of squamous tumours. a, Quantification of tumour weight of Bmal1WT/K5-SOS and Bmal1KO/K5SOS littermates $(n \geq 5)$. b, FACS analysis and quantification of $\alpha 6^{\text {bright }} / \mathrm{CD} 34^{+}$ tumour-initiating cells (P36 mice). Numbers represent percentage of $\alpha 6^{\text {bright }}$ / CD $34^{+}$cells from gated epithelial cells $(n \geq 3)$ c, Histological analysis and immunostaining for $\alpha 6$-integrin (arrows indicate suprabasal expression). Scale bars, $50 \mu \mathrm{m}$. Results in $\mathbf{a}$ and $\mathbf{b}$ are shown as mean \pm s.e.m., ${ }^{*} P<0.05$ (twotailed Student's $t$-test).

cells (Fig. $5 \mathrm{c}$ ). The reduction in the percentage of tumour-initiating cells, together with the high expression of several tumour suppressors, in Bmal1KO epidermal progenitors, including the non-coding RNA H19 (top upregulated gene; Supplementary Table 3), probably contribute to the reduced burden of squamous lesions in Bmal1 KO mice.

\section{Discussion}

Our results indicate that the molecular clock establishes stem cell states that are differentially predisposed to respond to activation and dormancy stimuli. The clock machinery controls the expression of essential epidermal stem cell regulators to anticipate the requirements of the tissue. In this sense, in the epidermal compartment where quiescent stem cells exist (that is, the bulge), the clock establishes a population of 'ready-to-go' cells that can rapidly and efficiently respond to activation stimuli, while simultaneously preventing all stem cells within the niche from becoming responsive. Future studies will be necessary to determine when, and how, this stem cell heterogeneity is established. On the other hand, the murine interfollicular epidermis, which primarily depends on continuously cycling basal progenitors rather than dormant stem cells ${ }^{38}$ for its renewal, shows a much more homogenous clock activity (albeit containing approximately $5 \%$ of cells antiphasic with the majority). In this compartment the clock machinery might predominantly establish a correct timing of stem cell activation and differentiation. Conditional deletion of Bmall in liver, retina and pancreas results in profound defects in tissue function ${ }^{39-41}$, and haematopoietic stem cells show a Bmal1/Clock-dependent circadian release to the periphery ${ }^{42}$. Altogether, these findings indicate that the clock machinery may constitute a fine-tuning homeostatic mechanism in tissues in which dormant and active populations of stem cells coexist. Unbalancing the epidermal stem cell clock not only substantially affected longterm tissue homeostasis, but also the predisposition of the tissue to undergo neoplastic transformation. It is likely that perturbations of this clock-controlled mechanism over stem cell regulation in humans may have long-term consequences on tissue homeostasis, ageing and carcinogenesis.

\section{METHODS SUMMARY}

For the isolation of epidermal cells from back or tail skin, the skin was incubated in $0.25 \%$ trypsin for $2 \mathrm{~h}$ at $37^{\circ} \mathrm{C}$, or overnight at $4{ }^{\circ} \mathrm{C}$, to separate the dermis from the epidermis. Back and tail keratinocytes were extracted as described previously ${ }^{43}$.

For ChIP assays, cells in suspension were cross-linked for $10 \mathrm{~min}$ at room temperature $\left(22-25^{\circ} \mathrm{C}\right)$ in $1 \%$ formaldehyde. Cross-linking reactions were stopped by adding $1.25 \mathrm{M}$ glycine to a final concentration of $125 \mathrm{mM}$. Cells were centrifuged at $300 \mathrm{~g}$ for $10 \mathrm{~min}$ at $4{ }^{\circ} \mathrm{C}$ and washed in cold PBS. Cell lysis, sonification and ChIP assays were performed using the MAGnify Chromatin Immunoprecipitation System (Invitrogen).

For FACS analysis or sorting of bulge and epidermal stem cells, cell suspensions were incubated for $30 \mathrm{~min}$ on ice with PE-conjugated anti- $\alpha 6$-integrin (CD49f clone NKI-GoH3, Serotec) and biotin-conjugated anti-CD34 (clone RAM34, BD Pharmingen) antibodies followed by APC-conjugated streptavidin (BD Pharmingen) for $20 \mathrm{~min}$. Dead cells were excluded by 4',6-diamidino-2phenylindole (DAPI) incorporation. FACS analysis and sorting were performed using LSRII FACS Analysers, FACSAriaII, FACSDiva (BD Biosciences) and Flowjo software.

For microarray analysis, total RNA was isolated from FACS-sorted cells using Trizol extraction and RNeasy Micro Kit (Qiagen). Transcriptional profiling was performed using GeneChip Mouse Gene 1.0 ST Array (Affymetrix) and functional analysis was performed using DAVID Bioinformatics Resources 6.7.

Full Methods and any associated references are available in the online version of the paper at www.nature.com/nature.

1. Fuchs, $E$. The tortoise and the hair: slow-cycling cells in the stem cell race. Cell 137, 811-819 (2009).

2. Morris, R. J. et al. Capturing and profiling adult hair follicle stem cells. Nature Biotechnol. 22, 411-417 (2004)

3. Tumbar, T. et al. Defining the epithelial stem cell niche in skin. Science $\mathbf{3 0 3}$, 359-363 (2004).

4. Jaks, V.etal. Lgr5 marks cycling, yet long-lived, hair follicle stem cells. Nature Genet 40, 1291-1299 (2008)

5. Snippert, H. J. et al. Lgr6 marks stem cells in the hair follicle that generate all cell lineages of the skin. Science 327, 1385-1389 (2010).

6. Jensen, K. B. et al. Lrig1 expression defines a distinct multipotent stem cell population in mammalian epidermis. Cell Stem Cell 4, 427-439 (2009).

7. Jensen, U. B. et al. A distinct population of clonogenic and multipotent murine follicular keratinocytes residing in the upper isthmus. J. Cell Sci. 121, 609-617 (2008).

8. Clayton, E. etal. A single type of progenitor cell maintains normal epidermis. Nature 446, 185-189 (2007).

9. Kobielak, K., Stokes, N., de la Cruz, J., Polak, L. \& Fuchs, E. Loss of a quiescent niche but not follicle stem cells in the absence of bone morphogenetic protein signaling. Proc. Natl Acad. Sci. USA 104, 10063-10068 (2007).

10. Guasch, G. et al. Loss of TGF $\beta$ signaling destabilizes homeostasis and promotes squamous cell carcinomas in stratified epithelia. Cancer Cell 12, 313-327 (2007).

11. Plikus, M. V. et al. Cyclic dermal BMP signalling regulates stem cell activation during hair regeneration. Nature 451, 340-344 (2008).

12. Zhang, Y. V., Cheong, J., Ciapurin, N., McDermitt, D. J. \& Tumbar, T. Distinct selfrenewal and differentiation phases in the niche of infrequently dividing hair follicle stem cells. Cell Stem Cell 5, 267-278 (2009).

13. Greco, V. et al. A two-step mechanism for stem cell activation during hair regeneration. Cell Stem Cell 4, 155-169 (2009).

14. Enshell-Seijffers, D., Lindon, C., Kashiwagi, M. \& Morgan, B. A. $\beta$-Catenin activity in the dermal papilla regulates morphogenesis and regeneration of hair. Dev. Cell 18, 633-642 (2010).

15. Brownell, I., Guevara, E., Bai, C. B., Loomis, C. A. \& Joyner, A. L. Nerve-derived sonic hedgehog defines a niche for hair follicle stem cells capable of becoming epidermal stem cells. Cell Stem Cell 8, 552-565 (2011).

16. Flores, I., Cayuela, M. L. \& Blasco, M. A. Effects of telomerase and telomere length on epidermal stem cell behavior. Science 309, 1253-1256 (2005)

17. Owens, D. M. \& Watt, F. M. Contribution of stem cells and differentiated cells to epidermal tumours. Nature Rev. Cancer 3, 444-451 (2003).

18. Watt, F. M., Frye, M. \& Benitah, S. A. MYC in mammalian epidermis: how can an oncogene stimulate differentiation? Nature Rev. Cancer 8, 234-242 (2008).

19. Benitah, S. A., Frye, M., Glogauer, M. \& Watt, F. M. Stem cell depletion through epidermal deletion of Rac1. Science 309, 933-935 (2005).

20. Morris, R. J. A perspective on keratinocyte stem cells as targets for skin carcinogenesis. Differentiation 72, 381-386 (2004)

21. Dibner, C., Schibler, U. \& Albrecht, U. The mammalian circadian timing system: organization and coordination of central and peripheral clocks. Annu. Rev. Physiol. 72, 517-549 (2010)

22. Cheng, H. Y. et al. Segregation of expression of mPeriod gene homologs in neurons and glia: possible divergent roles of mPeriod 1 and mPeriod 2 in the brain. Hum. Mol. Genet. 18, 3110-3124 (2009).

23. Kuhlman, S. J., Quintero, J. E. \& McMahon, D. G. GFP fluorescence reports period 1 circadian gene regulation in the mammalian biological clock. Neuroreport 11, 1479-1482 (2000)

24. Schneider, M. R., Schmidt-Ullrich, R. \& Paus, R. The hair follicle as a dynamic miniorgan. Curr. Biol. 19, R132-R142 (2009)

25. Bunger, M. K. et al. Mop3 is an essential component of the master circadian pacemaker in mammals. Cell 103, 1009-1017 (2000).

26. Lin, K. K. et al. Circadian clock genes contribute to the regulation of hair follicle cycling. PLoS Genet. 5, e1000573 (2009). 
27. Kondratov, R. V., Kondratova, A. A., Gorbacheva, V. Y., Vykhovanets, O. V. \& Antoch, M. P. Early aging and age-related pathologies in mice deficient in BMAL1, the core componentof the circadian clock. Genes Dev. 20, 1868-1873 (2006).

28. Kameda, T. \& Sugiyama, T. Application of genetically modified feeder cells for culture of keratinocytes. Methods Mol. Biol. 289, 29-38 (2005).

29. Zheng, B. et al. Nonredundant roles of the mPer1 and mPer2 genes in the mammalian circadian clock. Cell 105, 683-694 (2001).

30. Barradas, M. et al. Histone demethylase JMJD3 contributes to epigenetic control of INK4a/ARF by oncogenic RAS. Genes Dev. 23, 1177-1182 (2009).

31. Ito, M. etal. Stem cells in the hair follicle bulge contribute to wound repair but not to homeostasis of the epidermis. Nature Med. 11, 1351-1354 (2005).

32. Gatfield, D. et al. Integration of microRNA miR-122 in hepatic circadian gene expression. Genes Dev. 23, 1313-1326 (2009).

33. Rogler, C. E. et al. MicroRNA-23b cluster microRNAs regulate transforming growth factor- $\beta$ /bone morphogenetic protein signaling and liver stem cell differentiation by targeting Smads. Hepatology 50, 575-584 (2009).

34. Fu, L. \& Lee, C. C. The circadian clock: pacemaker and tumour suppressor. Nature Rev. Cancer 3, 350-361 (2003)

35. Sibilia, M. et al. The EGF receptor provides an essential survival signal for SOSdependent skin tumor development. Cell 102, 211-220 (2000).

36. Malanchi, I. et al. Cutaneous cancer stem cell maintenance is dependent on $\beta$-catenin signalling. Nature 452, 650-653 (2008).

37. Owens, D. M., Romero, M. R., Gardner, C. \& Watt, F. M. Suprabasal $\alpha 6 \beta 4$ integrin expression in epidermis results in enhanced tumourigenesis and disruption of TGF $\beta$ signalling. J. Cell Sci. 116, 3783-3791 (2003).

38. Clayton, E. et al. A single type of progenitor cell maintains normal epidermis. Nature 446, 185-189 (2007)

39. Storch, K. F. etal. Intrinsic circadian clock of the mammalian retina: importance for retinal processing of visual information. Cell 130, 730-741 (2007).

40. Marcheva, B. etal. Disruption of the clock components CLOCK and BMAL1 leads to hypoinsulinaemia and diabetes. Nature 466, 627-631 (2010).
41. Lamia, K. A., Storch, K. F. \& Weitz, C. J. Physiological significance of a peripheral tissue circadian clock. Proc. Natl Acad. Sci. USA 105, 15172-15177 (2008).

42. Méndez-Ferrer, S., Lucas, D., Battista, M. \& Frenette, P. S. Haematopoietic stem cell release is regulated by circadian oscillations. Nature 452, 442-447 (2008).

43. Jensen, K. B., Driskell, R. R. \& Watt, F. M. Assaying proliferation and differentiation capacity of stem cells using disaggregated adult mouse epidermis. Nature Protocols 5, 898-911 (2010).

Supplementary Information is linked to the online version of the paper at www.nature.com/nature.

Acknowledgements We thank the AICR (Association for International Cancer Research), the Spanish Ministry of Health (FIS) and AGAUR (Agència de Gestió d'Ajuts Universitaris i de Recerca; Government of Cataluña) for financial support. P.J. is the recipient of an AGAUR PhD Fellowship, and G.P. of a FIS fellowship. We thank D. McMahon (Vanderbilt University) for providing us with the Per1-GFP mice; E. Wagner (CNIO) for the K5-SOS mice; B. Kübler, the FACS and Genomics units of the IRB (Institute de Recerca Biomedica), the CRG (Center for Genomic Regulation) core facilities and the Animal Unit (Juan Martin Caballero) for technical support.

Author Contributions P.J. performed the experiments, and P.J. and S.A.B. analysed the results and wrote the manuscript. G.P. performed the analysis of K5-SOS mice, and A.M. and E.B. assisted P.J. in the initial FACS sorts. L.D.C. helped us with the initial ChIP experiments. K.O. provided the Per1-venus mice. J.R. and U.A. provided the Per1/ Per2dKO mice.

Author Information Microarray data can be retrieved from the Gene Expression Omnibus under accession number GSE27079. Reprints and permissions information is available at www.nature.com/reprints. The authors declare no competing financial interests. Readers are welcome to comment on the online version of this article at www.nature.com/nature. Correspondence and requests for materials should be addressed to S.A.B. (salvador.aznar-benitah@crg.es). 


\section{METHODS}

Animals. Bmal1 ${ }^{\text {LoxP/LoxP }}$ and K14Cre mice purchased from The Jackson Laboratory were crossed with each other to obtain $\mathrm{K} 14 \mathrm{Cre} / \mathrm{Bmal1}{ }^{\mathrm{wt} / \mathrm{wt}}$ (Bmal1WT) and K14Cre/Bmal1 ${ }^{\text {LoxP/LoxP }}$ (Bmal1KO), and with K5-SOS mice to generate $\mathrm{K} 14 \mathrm{Cre} / \mathrm{Bmal1}{ }^{\mathrm{wt} / \mathrm{wt}} / \mathrm{K} 5-\mathrm{SOS}$ (Bmal1WT/K5SOS) and $\mathrm{K} 14 \mathrm{Cre} /$ Bmal1 ${ }^{\text {LoxP/LoxP } / K 5-S O S ~(B m a l 1 K O / K 5 S O S) ~ l i t t e r m a t e ~ c o n t r o l s ~}{ }^{35}$. Per1-GFP and Perl-venus mice have been described previously ${ }^{22,23}$. K14Cre/Bmal1 ${ }^{\mathrm{wt} / \mathrm{wt}}$ Per1-venus and K14Cre/Bmal1 ${ }^{\text {LoxP/LoxP }}$ Perl-venus mice were generated by crossing $\mathrm{K} 14 \mathrm{Cre} / \mathrm{Bmal1} 1^{\mathrm{wt} / \mathrm{wt}}$ and $\mathrm{K} 14 \mathrm{Cre} / \mathrm{Bmal1}{ }^{\mathrm{LoxP} / \mathrm{LoxP}}$ animals with Per1venus mice, respectively. Per 1 and Per 2 double-mutant mice (Per1/2dKO) have been described previously, and were compared to age and sex-matched control animals to avoid differences in hair cycle behaviour between males and females ${ }^{29}$. Mice were housed in an AAALAC-I approved animal unit under $12 \mathrm{~h}$ light $/ 12 \mathrm{~h}$ dark or $12 \mathrm{~h}$ dark $/ 12 \mathrm{~h}$ dark cycles, and SPF conditions, and all procedures were approved by the CEEA (Ethical Committee for Animal Experimentation) of the Government of Catalonia. For experiments in constant darkness, light was turned off at Zeitgeber time (ZT)12 and animals were housed in $12 \mathrm{~h}$ dark/12 h dark conditions for 5 days. For 5-bromo-2-deoxyuridine (BrdU)-labelling experiments, $100 \mathrm{\mu g} \mathrm{g}^{-1} \mathrm{BrdU}$ (Invitrogen) was injected intraperitoneally into the mice and chased for 10 weeks or up to 10 months, as indicated. To activate epidermal proliferation, back skin or tail skin of 10-week-old Bmal1WT and BmallKO mice was treated three times with 20 nM TPA (Sigma-Aldrich) during one week. For depilation experiments, dorsal skin of 15-month-old Bmal1WT and Bmal1KO mice in telogen phase was depilated with cold wax strips (Taky).

Primary mouse keratinocyte cultures. Primary mouse keratinocytes from newborn mice or tail skin of adult mice were isolated as described previously ${ }^{43,44}$. Cells were plated in EMEM (Lonza) containing 4\% chelated FBS, $1 \%$ penicillin/ streptomycin and $20 \mathrm{nM}$ calcium for $24 \mathrm{~h}$; medium was then changed to growth medium (EMEM with $4 \%$ chelated FBS, $1 \%$ penicillin/streptomycin, EGF (10 ng $\mathrm{ml}^{-1}$ ) and $50 \mathrm{nM}$ calcium). For the time-course experiments of keratinocytes isolated from Bmal1WT or Bmal1 KO mice, cells were synchronized by a serum shock with growth medium, containing $50 \%$ chelated FBS, for $2 \mathrm{~h}^{45}$. For TGF- $\beta$ treatments, keratinocytes were cultured in the presence of TGF- $\beta 1\left(2 \mathrm{ng} \mathrm{ml}^{-1}\right.$; PreproTech) for $48 \mathrm{~h}$. For clonogenic assays, the indicated number of FACSsorted keratinocytes were plated in E-medium on mitomycin (Sigma-Aldrich)treated J2 3T3 feeder cells ${ }^{46}$. Keratinocytes were grown at $37^{\circ} \mathrm{C}$ with $5 \% \mathrm{CO}_{2}$. Whole-mount immunofluorescence. Preparation of tail skin and whole-mount stainings were performed as previously described ${ }^{47}$. Primary and secondary antibodies were incubated overnight and used at the following concentrations: 1:1,000 for anti-GFP (A11122, Invitrogen); 1:250 for anti-BrdU (Serotec) and anti-Ki67 (ab15580, Abcam; clone B56, BD Pharmingen); and 1:500 for antirabbit and anti-rat conjugated to AlexaFluor488 or AlexaFluor594 (Molecular Probes). Nuclei were stained with DAPI (1:5000; Roche), and epidermal sheets were mounted in Mowiol. Pictures were acquired with a Leica TCS SP5 confocal microscope.

Immunohistochemistry. Back skin and tail skin were either embedded in OCT, or fixed in $4 \% \mathrm{NBF}$ (Sigma-Aldrich) at $4{ }^{\circ} \mathrm{C}$ overnight or $2 \mathrm{~h}$ at room temperature $\left(22-25^{\circ} \mathrm{C}\right)$ and then embedded in paraffin or OCT. Deparaffinized sections were boiled for $10 \mathrm{~min}$ in $0.01 \mathrm{M}$ citric acid for antigen retrieval. For permeabilization, 8 - $\mu \mathrm{m}$ slices of either cryosections, or sections that had been deparaffinized or antigen retrieved were permeabilized for $25 \mathrm{~min}$ in $0.25 \%$ Triton X-100/PBS and blocked for $90 \mathrm{~min}$ in $0.25 \%$ gelatin/PBS. Primary antibodies were incubated overnight at $4{ }^{\circ} \mathrm{C}$, and secondary antibodies were incubated for $2 \mathrm{~h}$ at room temperature in $0.25 \%$ gelatin/PBS. Nuclei were stained with DAPI $(1: 5,000$, Roche), and the slides were mounted in Mowiol. Primary antibodies were used at the following dilutions: 1:50 for anti-p19 (M-167, Santa Cruz), anti-p16 (M-156, Santa Cruz); 1:100 for anti-Sox9 (H-90, Santa Cruz); 1:200 for antia6-integrin (CD49f clone NKI-GoH3, Serotec), anti-keratin 14 (ab7800, Abcam); 1:250 for anti-Ki67 (ab15580, Abcam); 1:500 for anti-keratin 5 (ab24647, Abcam); and 1:1,000 for anti-GFP (ab290, Abcam; A11122, Invitrogen), anti-involucrin (PRB-140C, Covance), anti-filaggrin (ab24584, Abcam), anti-loricrin (PRB-145P, Covance), anti-phospho-Smad2 (\#3101, Cell Signaling), anti-Lef1 (clone C12A5, Cell Signaling). Secondary antibodies were used at dilutions of 1:500: anti-rabbit, anti-mouse, or anti-rat, conjugated to AlexaFluor488 or AlexaFluor594 (Molecular Probes), or anti-rabbit biotin (GE Healthcare), followed by an incubation with ABC Kit (Vector Laboratories) and FAST 3,3 Diaminobenzidine tablets (Sigma-Aldrich). For apoptosis measurements, tail skin was stained with the DeadEnd Fluorometric TUNEL System (Promega). Staining with haematoxilin or eosin was done according to a standard protocol. Pictures were acquired with a Leica DMI 6000B or a Leica TCS SP5 confocal microscope.

Time-lapse microscopy and quantification. For time-lapse confocal imaging, back skin of Perl-venus mice was fixed with $0.5 \%$ agarose in an imaging dish
(Ibidi) and over-layered with E-medium. Images were taken every $15 \mathrm{~min}$ for a period of $48 \mathrm{~h}$ using a Leica TCS SP5 confocal microscope equipped with a tempered chamber of $37^{\circ} \mathrm{C}$ and $5 \% \mathrm{CO}_{2}$. Mean fluorescence intensity of individual GFP-positive nuclei was quantified using ImageJ software.

FACS. Epidermal cells from back skin of Per1-venus, Bmal1WT and Bmal1KO mice, or tail skin of Bmal1WT/K5-SOS and Bmal1KO/K5-SOS mice, were isolated as described previously ${ }^{43}$. Per1-venus mice were killed between ZT10-ZT12 or as specified in the figure legends. Cell suspensions were incubated for $30 \mathrm{~min}$ on ice with the following antibodies at the given dilutions: 1:100 for biotin- or APCconjugated anti-CD34 (clone RAM34, BD Pharmingen), PE-conjugated antiCD31 (clone MEC13.3, eBioscience), PE-conjugated anti-CD45 (clone 30F11, eBioscience), PE-conjugated anti-CD140a (clone APA5, BD Pharmingen) and APC-Cy7-conjugated anti-epcam (clone G8.8, Biolegend); 1:200 for PE- or FITC-conjugated anti- $\alpha 6$-integrin (CD49f clone NKI-GoH3, Serotec); and 1:500 for APC-conjugated streptavidin (BD Pharmingen). DAPI staining was used to rule out that dead cells were present. FACS was performed using FACSAriaII and FACSDiva software (BD Biosciences). Sorted cells were collected in E-medium, either plated in culture or re-suspended in Trizol (Invitrogen), and subjected further to RNA isolation. FACS analysis was performed using LSRII FACS Analysers (BD Biosciences) and Flowjo software.

Arrays. Total RNA was isolated from FACS-sorted cells in Trizol by chloroform extraction, followed by the RNA clean-up protocol of RNeasy Micro or Mini Kit (Qiagen). Transcriptional profiling was performed using GeneChip Mouse Gene 1.0 ST Array (Affymetrix). Arrays of venus ${ }^{\text {bright }}$ and venus ${ }^{\text {dim }}$ bulge cells of P19 Per1-venus mice were performed as triplicates from a pool of $n=64$ mice. Arrays of epidermal cells from 10-months-old Bmal1WT and BmallKO mice were performed as triplicates using three independent mice in each group. Functional analysis of microarray data was performed using DAVID Bioinformatics Resources 6.7.

RT-qPCR. Total RNA from cultured or FACS-sorted cells was purified as described above, or using either the RNeasy Micro and Mini Kit (Qiagen) or miRVana miRNA isolation Kit (Ambion). Equal amounts of RNA were reverse-transcribed using Superscript III (Invitrogen). RT-qPCR was performed with SYBR Green Master Mix (Roche) and gene-specific primers (as given in Supplementary Table 4$)^{48}$ using a Light Cycler 480 Instrument (Roche). Relative levels of expression were determined by normalization to pumilio 1 (Pum1), using the $\Delta \Delta C_{\mathrm{t}}$ method.

ChIP. For ChIP assays from intact epidermis, mice were killed at ZT2, or for time-course analysis every $3 \mathrm{~h}$ during a period of $24 \mathrm{~h}$, and tails were incubated in $0.25 \%$ trypsin for $2 \mathrm{~h}$ at $37^{\circ} \mathrm{C}$ to separate the dermis from the epidermis. Tail keratinocytes were extracted as described ${ }^{43}$. Cells in suspension or FACS-purified cells $\left(2 \times 10^{5}\right)$ were cross-linked for $10 \mathrm{~min}$ at room temperature in $1 \%$ formaldehyde. Cross-linking reactions were stopped by adding $1.25 \mathrm{M}$ glycine to a final concentration of $125 \mathrm{mM}$. Cells were centrifuged at $300 \mathrm{~g}$ for $10 \mathrm{~min}$ at $4{ }^{\circ} \mathrm{C}$ and washed in cold PBS. Cell lysis, sonification and ChIP assays were performed using the MAGnify Chromatin Immunoprecipitation System (Invitrogen). For each immunoprecipitation, $2 \times 10^{5}-10^{6}$ cells were incubated with $2 \mu \mathrm{l}$ of anti-Bmall or anti-Clock antibody (provided by J. Ripperger, University of Fribourg) ${ }^{49}$, or $2 \mu$ l of rabbit IgG control antibody (Invitrogen). $\mathrm{RT}-\mathrm{qPCR}$ was performed as described above using gene-specific primers (Supplementary Table 5).

Genotyping and western blots. Genomic DNA of primary mouse keratinocytes from Bmal1WT and Bmal1KO mice was isolated using a standard protocol. Multiplex PCR was performed as previously described to determine recombination efficiency ${ }^{39}$. Protein extracts from newborn keratinocytes or from tail epidermis were analysed by SDS-PAGE and western blotting for Bmal1 (ref. 49), anti-phospho-Smad2 (1:1,000, \#3101, Cell Signaling), anti-Lef1 (1:1,000, clone C12A5, Cell Signaling), anti-Sox9 (1:400, H-90, Santa Cruz) and anti-tubulin (1:5,000, Sigma Aldrich)

Luciferase assay. Primary mouse keratinocytes were transiently transfected with either FOP-Flash or TOP-Flash and pCMV-Renilla plasmids using FuGENE6 (Roche) according to the manufacturer's instructions. The TOP-Flash construct reports Wnt activity by driving the expression of Luciferase under the regulation of TCF-Lef binding sites. FOP-Flash contains the same binding sites mutated so that they are no longer responsive to TCF/Lef. Cells were treated in the absence or presence of $1 \mu \mathrm{M}$ BIO (Calbiochem) at $48 \mathrm{~h}$ post-transfection for a period of $24 \mathrm{~h}$. Luciferase activity was measured in a Centro LB 960 luminometer (Berthold Technologies) using Dual-Luciferase Reporter Assay System (Promega).

Promoter analysis. Gene promoter analysis for potential Bmall/Clock-binding sites (in general, from $-5,000$ to $+1,000$ bases from the transcriptional start site) were analysed using Genomatix Software.

Statistics. Results are presented as mean \pm s.e.m. Statistical significance was determined by two-tailed Student's $t$-test, one-way ANOVA, two-way ANOVA 
with Bonferroni post-test, or Cosinor analysis. A $P$ value of $P \leq 0.05$ was considered to be statistically significant.

44. Litchi, U., Anders, J. \& Yuspa, S. Isolation and short-term culture of primary keratinocytes, hair follicle populations and dermal cells from newborn mice and keratinocytes from adult mice for in vitro analysis and for grafting to immunodeficient mice. Nature Protocols 5, 799-810 (2008).

45. Balsalobre, A., Damiola, F. \& Schibler, U. A serum shock induces circadian gene expression in mammalian tissue culture cells. Cell 93, 929-937 (1998).
46. Nowak, J. A. \& Fuchs, E. Isolation and culture of epithelial stem cells. Methods Mol. Biol. 482, 215-232 (2009).

47. Braun, K. M. et al. Manipulation of stem cell proliferation and lineage commitment: visualization of label-retaining cells in wholemounts of mouse epidermis. Development 130, 5241-5255 (2003)

48. Sun, F. et al. Characterization of function and regulation of miR-24-1 and miR-31. Biochem. Biophys. Res. Commun. 380, 660-665 (2009).

49. Ripperger, J. \& Schibler, U. Rhythmic CLOCK-BMAL1 binding to multiple E-box motifs drives circadian $D b p$ transcription and chromatin transitions. Nature Genet. 38, 369-374 (2006) 